\title{
Modelado de un Sistema de Evaporación de Múltiple Efecto para la Producción de Panela (Azúcar no Centrifugado)
}

\author{
Roger A. Ordoñez, Cesar A. Hernández* y Luis F. Pedraza \\ Universidad Distrital "Francisco José de Caldas", Grupo de Investigación Internet Inteligente, \\ Carrera 7 No. 40 - 53 Bogotá-Colombia. \\ (e-mail: \{raordonez, cahernandezs, Ifpedrazam\}@udistrital.edu.co\})
}

${ }^{*}$ Autor a quien debe ser dirigida la correspondencia.

Recibido Mar. 19, 2012; Aceptado May. 24, 2012; Versión final recibida Ago. 07, 2012

\section{Resumen}

Se propone un método para optimizar los tiempos de procesamiento y los recursos en la industria de la panela, azúcar no centrifugado, cuyo único ingrediente es el jugo de la caña de azúcar. La metodología consistió en modelar el sistema de evaporización de múltiple efecto utilizando datos experimentales de un sistema real de evaporización de tres efectos y que permitiera controlar la concentración de azúcar y el nivel del fluido, en cada efecto. Como resultado se obtuvo un modelo del sistema de evaporización de múltiple efecto mediante una función de transferencia de tercer orden y un control proporcional derivativo. Finalmente se muestra que el control de la concentración de azúcar y el nivel del fluido en cada efecto permiten obtener un sistema de evaporación de múltiple efecto más eficiente en términos de tiempos de procesamiento y optimización de recursos.

\section{Modeling of a Multiple Effect Evaporation System for the Production of Panela (Non-Centrifuged Sugar)}

\begin{abstract}
A method for optimizing processing times and resources in the production of panela, noncentrifuged sugar, with sugar cane juice as the single ingredient. The methodology consisted of modeling a multiple effect evaporation system using experimental data from a real system of three effects that allowed controling the concentration of sugar and the liquid level in each effect. As results, a model system of multiple effect evaporation through a transfer function of third order and a proportional-derivative control were obtained. Finally, it is shown that controling the sugar concentration and the level of liquid in each effect allow to obtain a system of multiple effect evaporation that is more efficient in terms of processing time and optimization of resources.
\end{abstract}

Keywords: concentration, control, evaporation, panela, thermodynamic, sugar cane 


\section{INTRODUCCIÓN}

La panela es definida por la Organización de las Naciones Unidas para la agricultura y la alimentación -FAO- como azúcar no centrifugado, cuyo único ingrediente es el jugo de la caña de azúcar. Los principales productores de panela a nivel mundial son India con aproximadamente el $86 \%$ del mercado y Colombia con alrededor del $13.9 \%$ del mercado que equivale a 1.4 millones de toneladas anuales.

El proceso panelero en general consiste en moler la caña, separar las impurezas, calentar el jugo, descachazar, evaporar hasta el punto de panela, realizar el batido y finalmente el envasado en unos moldes en forma de cubo donde se deja secar hasta que se solidifica. El proceso se lleva a cabo en un lugar denominado trapiche, en donde se localiza el molino y las hornillas y es allí donde se concentran los sólidos solubles totales que existen en el jugo de la caña de azúcar, todo ello mediante la transferencia térmica en las hornillas y llevando a cabo la concentración de los jugos (Rodríguez, 2004; Rodríguez 2008).

Según la experiencia del Centro Internacional de Mejoramiento de la Panela (CIMPA), la tecnología de la producción de panela no ha variado a través de los años, ya que el producto se obtiene fundamentalmente como resultado de la evaporación del jugo de caña, por tanto ha sido poco tecnificado y optimizado.

En Colombia se hacen inversiones de infraestructura para el mejoramiento tecnológico en el proceso de elaboración de la panela a través de los trapiches a vapor, acortando la brecha tecnológica con otros productores. Sin embargo, aún existen procesos que pueden ser mejorados permitiendo optimizar los recursos.

La gran mayoría de estudios, investigaciones y ajustes de tecnología sobre la producción de panela se centran en la generaron de diversos diseños de equipos y recomendaciones de manejo del cultivo y del proceso, los cuales pueden ser clasificados en: 1) recomendaciones agronómicas para el cultivo de la caña, 2) recomendaciones para el procesamiento de la caña y 3) recomendaciones para el aprovechamiento de los subproductos del proceso (Gravois et al., 2010).

En la actualidad, el proceso panelero es realizado mediante una evaporación abierta, generando desventajas energéticas de recursos naturales y de productividad; por esto es necesario realizar un cambio tecnológico que aumente la baja competitividad existente de los pequeños y medianos productores (Mosquera et al., 2005; Duran, 2010).

Un evaporador consiste básicamente de un intercambiador de calor capaz de hervir la solución y un dispositivo para separar la fase vapor del líquido en ebullición (Rodríguez, 2008). Los sistemas de evaporadores industriales normalmente constan de a) un intercambiador de calor para la evaporación de la sustancia a concentrar (en la industria de los alimentos normalmente se utiliza como medio de calentamiento vapor saturado); b) un separador en el que el vapor se aparta de la fase líquida concentrada (en los sistemas que operan a presión atmosférica el separador puede omitirse); y c) Un condensador, para condensar el vapor y eliminar el agua condensada del sistema (Rodríguez y Requierdesjardins, 2004; Bischoff y Gravois, 2003).

Durante la ebullición, a medida que aumenta la concentración de la solución, propiedades como la densidad y la viscosidad aumentan con el contenido de sólidos, hasta que la solución se transforma en saturada o resulta inadecuada para una correcta transferencia de calor (García et al., 2009).

En la evaporación de múltiple efecto, el vapor vivo procedente de la caldera se introduce a un primer evaporador (primer efecto) como alimentación en el elemento calefactor, y el vapor producido durante este efecto, se introduce como alimentación al siguiente evaporador (segundo efecto), y así sucesivamente hasta el último efecto, en donde el vapor producido se envía a un condensador (Nielsen et al., 1996). 
La presión en cada efecto es menor que la del efecto anterior, del cual recibe el vapor, y superior a la del efecto al cual suministra el vapor. Cada efecto por sí solo actúa como un evaporador de un sólo efecto, y en cada uno de ellos hay una reducción en la temperatura de ebullición correspondiente a la caída de presión en dicho efecto (Rahman et al., 2009).

El principal aporte y contribución del presente artículo al conocimiento actual en el desarrollo tecnológico de la agroindustria de la panela, es el mejoramiento de los tiempos de procesamiento y la optimización de recursos, mediante el modelado de un sistema de evaporación de tres efectos y el aprovechamiento del vapor en sistemas cerrados, del ahorro energético y de la termodinámica inmersa en el sistema (Nielsen et al., 1996).

\section{RECONOCIMIENTO DEL SISTEMA}

\section{Ley de Antoine}

Al reducir la temperatura de ebullición del líquido, aumenta la diferencia de temperatura entre el vapor y el líquido en ebullición, y por tanto, aumenta la velocidad de transferencia de calor en el evaporador, este fenómeno se describe por la "Ley de Antoine" Ec. (1), en donde, se establece una relación matemática que permite conocer la temperatura de ebullición de un líquido a diferentes presiones.

$\log \mathrm{P}=\mathrm{A}+\frac{\mathrm{B}}{\mathrm{C}+\mathrm{T}}$

Donde:

$\mathrm{P}=$ Presión de vapor $(\mathrm{Pa})$

$\mathrm{A}, \mathrm{B}, \mathrm{C},=$ Constantes experimentales del líquido

$\mathrm{T}=$ Temperatura de ebullición $\left({ }^{\circ} \mathrm{C}\right)$

\section{Trasferencia de Calor}

Para concentrar una sustancia, en este caso los jugos de caña, se realiza una evaporación de tal manera que se reduzca el porcentaje de agua y otras sustancias que se convierten en vapor, quedando en la sustancia que ebulle los jugos de caña más concentrados. Este proceso se realiza por intercambio de calor convectivo, al realizar el intercambio el volumen de los jugos iníciales disminuye por la evaporación que se realiza, la sustancia que suministra este calor es el vapor de caldera. Cuando el vapor de caldera entra al primer efecto se realiza el intercambio de calor, el cual se describe por la Ec. (2) (Ruiz, 2011).

$\mathrm{Q}=\mathrm{UA} \Delta \mathrm{T}$

Donde:

$\mathrm{Q}=$ Intercambio de calor $(\mathrm{W})$

$\mathrm{U}=$ coeficiente global de transferencia de calor $\left(\mathrm{W} /{ }^{\circ} \mathrm{C} \mathrm{m}^{2}\right)$

$\mathrm{A}=$ Área $\left(\mathrm{m}^{2}\right)$

$\Delta \mathrm{T}=$ Diferencia de temperatura entre las dos sustancias $\left({ }^{\circ} \mathrm{C}\right)$

El intercambio de calor por convección puede ser latente o sensible. El calor sensible descrito por la Ec. (3), hace referencia al calor que recibe un cuerpo, provocando el aumento de su temperatura sin afectar su estructura molecular y por tanto su estado. El calor latente descrito por la Ec. (4), es la energía requerida por una sustancia para cambiar de fase o estado.

$$
\mathrm{Q}=\mathrm{WC} \mathrm{C}_{\mathrm{p}} \Delta \mathrm{T}
$$

Donde:

$\mathrm{Q}=$ Intercambio de calor $(\mathrm{J})$

$\mathrm{C}_{\mathrm{p}}=$ Calor específico $\left(\mathrm{J} / \mathrm{kg}^{\circ} \mathrm{C}\right)$ 
$\Delta \mathrm{T}=$ Diferencia de temperatura entre dos sustancias $\left({ }^{\circ} \mathrm{C}\right)$

$\mathrm{W}=$ masa $(\mathrm{kg})$

\section{$\mathrm{Q}=(\mathrm{W}$}

Donde:

$Q=$ Intercambio de calor $(\mathrm{J})$

$\lambda=$ Calor latente de evaporación $(\mathrm{J} / \mathrm{kg})$

$W=$ Masa $(\mathrm{kg})$

\section{Control de Nivel}

Cuando se habla de un control de nivel, indirectamente se hace referencia a un control de fluido, que se puede relacionar con circuito eléctrico haciendo la equivalencia de corriente eléctrica a la entrada del fluido, y la diferencia de potencial en una diferencia de presión $\left(P_{1}-P_{2}\right)$. Para este caso la resistencia en el sistema está representada por la resistencia del fluido a través de una válvula o el cambio de diámetro de las tuberías, la relación entre el flujo $Q$ a través de la resistencia $R$ es una diferencia de presión descrita por la Ec. (5), denominada resistencia hidráulica.

$$
\mathbf{P}_{1}-\mathbf{P}_{2}=\mathrm{RQ}
$$

Donde:

$\mathrm{P}=$ Presión $(\mathrm{N} / \mathrm{m})$

$\mathrm{R}=$ Resistencia hidráulica $\left(\frac{\mathrm{kg}}{\mathrm{m}^{4} \mathrm{~s}}\right)$

$\mathrm{Q}=$ Caudal $\left(\frac{\mathrm{m}^{3}}{\mathrm{~s}}\right)$

La capacitancia hidráulica descrita por la Ec. (6) es un término que se utiliza para describir el almacenamiento de energía de un líquido en forma de energía potencial, por tanto, la variación de volumen en un recipiente, es función de la diferencia de caudales que entran y salen.

$\mathrm{Q}_{1}-\mathrm{Q}_{2}=\frac{\mathrm{dV}}{\mathrm{dt}}$

Como el volumen $(V)$ se puede calcular mediante $V=A^{*} h$, donde $A$ es el área de la sección transversal del contenedor y $h$ la altura del fluido en él, la Ec. (6) se transforma en la Ec. (7):

$\mathrm{Q}_{1}-\mathbf{Q}_{2}=\frac{\mathrm{dV}}{\mathrm{dt}}=\mathrm{A} \frac{\mathrm{dh}}{\mathrm{dt}}$

Ahora, la presión se puede expresar en términos de la altura como lo muestra la Ec. (8):

$\mathrm{P}=\rho g h$

Donde $\rho$ es la densidad del líquido y $g$ es la aceleración de la gravedad, luego remplazando $\mathrm{h}$ de la Ec. (8) en la Ec. (7) se obtiene la Ec. (9).

$\mathrm{Q}_{1}-\mathrm{Q}_{2}=\frac{\mathrm{d}(\mathrm{P} / \mathrm{\rho g})}{\mathrm{dt}}=\frac{\mathrm{A}}{\mathrm{Pg}} \frac{\mathrm{dP}}{\mathrm{dt}}$

Debido a que el líquido es incomprensible, su densidad no cambia con la presión, la capacitancia $C$ se define en la Ec. (10): 
$\mathrm{C}=\frac{\mathrm{A}}{\rho \mathrm{g}}$

Remplazando (10) en (9) se obtiene la Ec. (11) para la capacitancia hidráulica:

$Q_{1}-Q_{2}=C \frac{d p}{d t}$

Donde:

$Q=$ Caudal $\left(\frac{\mathrm{m}^{3}}{\mathrm{~s}}\right)$

$C$ = Capacitancia $\left(\frac{\mathrm{m}^{2}}{\mathrm{~kg} / \mathrm{m}^{2} \mathrm{~s}^{2}}\right)$

$P=$ Presión $(\mathrm{N} / \mathrm{m})$

La inductancia hidráulica es el equivalente a un resorte en sistemas mecánicos, para acelerar el fluido e incrementar su velocidad se requiere una fuerza. Considerando un bloque de líquido de masa $m$ la fuerza neta que actúa sobre el líquido se determina por la Ec. (12).

$F_{1}-F_{2}=P_{1} A-P_{2} A=\left(P_{1}-P_{2}\right) A$

Donde $\left(P_{1}-P_{2}\right)$ es la diferencia de presiones y $A$ es el área de sección transversal. Como la fuerza neta es proporcional al producto masa por aceleración, la Ec. (12) se transforma en la Ec. (13).

$\left(\mathrm{P}_{1}-\mathrm{P}_{2}\right) \mathrm{A}=\mathrm{ma}$

Remplazando en la Ec. (13), a por la tasa de cambio de la velocidad $\mathrm{dV} / \mathrm{dt}$ y la masa del líquido por el producto volumen $A L$, donde $L$ es la longitud del bloque de líquido o la distancia entre los puntos donde se mide las presiones $P_{1}$ y $P_{2}$, y la densidad del liquido $\rho$, se obtiene la Ec. (14).

$\left(\mathbf{P}_{1}-\mathrm{P}_{2}\right) \mathrm{A}=\mathrm{AL} \rho \frac{\mathrm{dV}}{\mathrm{dt}}$

Remplazando la razón de flujo volumétrico $Q=A V$, se obtiene la Ec. (15).

$\left(\mathbf{P}_{1}-\mathbf{P}_{2}\right) \mathrm{A}=\mathrm{L} \boldsymbol{\rho} \frac{\mathrm{dQ}}{\mathrm{dt}}$

Definiendo la inductancia eléctrica como en la Ec. (16):

$\mathrm{I}=\frac{\mathrm{L} \rho}{\mathrm{A}}$

Y remplazando la Ec. (16) en la Ec. (15), se obtiene la Ec. (17) para la inductancia hidráulica:

$\left(\mathbf{P}_{1}-\mathbf{P}_{2}\right)=\mathrm{I} \frac{\mathrm{dQ}}{\mathrm{dt}}$

Donde:

$P=$ Presión $(\mathrm{N} / \mathrm{m})$

$I=$ Inductancia hidráulica $\left(\frac{\mathrm{kg}}{\mathrm{m}^{4}}\right)$

$Q=$ Caudal $\left(\frac{\mathrm{m}^{3}}{\mathrm{~s}}\right)$ 


\section{METODOLOGÍA}

Las variables que intervinieron en el modelado del sistema de evaporación de múltiple efecto fueron el caudal de entrada a cada efecto, la presión en cada efecto, la temperatura en cada efecto, el área de intercambio de cada efecto, la altura del fluido en cada efecto, los coeficientes globales de transferencia de calor en cada efecto, las entalpías de vaporización y el volumen de cada efecto, de acuerdo con el estudio realizado por (Zdravkovic et al., 1994; Duran, 2010) donde se resuelve el control de una estación de evaporización de efecto múltiple a partir de un control difuso PI.

Para realizar el modelado del sistema de evaporación de múltiple efecto se consideraron condiciones ideales tales como: la perdida nula de intercambio de calor con el ambiente y de la caída de presión en los efectos, despreciable desgaste de la tubería a través del tiempo y la cero formación de espuma en los jugos de caña durante la evaporación (Jose, 2011).

Además de esto, se tuvieron en cuenta datos de las variables, tomados experimentalmente y calculados previamente. En la Tabla 1 se presentan los valores utilizados para el Modelado.

Tabla 1. Datos experimentales para el Modelado del sistema de evaporación de múltiple efecto [8]

\begin{tabular}{|c|c|c|c|c|c|c|c|c|c|c|c|c|c|}
\hline Trat. & $\begin{array}{l}\text { Ws } \\
(\mathrm{kg} / \mathrm{h})\end{array}$ & $\begin{array}{c}W_{1} \\
(\mathrm{~kg} / \mathrm{h})\end{array}$ & $\begin{array}{c}W_{2} \\
(\mathrm{~kg} / \mathrm{h})\end{array}$ & $\begin{array}{c}W_{3} \\
(\mathrm{~kg} / \mathrm{h})\end{array}$ & $\begin{array}{c}\mathrm{Wa} \\
(\mathrm{kg} / \mathrm{h})\end{array}$ & $\begin{array}{l}W b \\
(\mathrm{~kg} / \mathrm{h})\end{array}$ & $\begin{array}{c}W c \\
(\mathrm{~kg} / \mathrm{h})\end{array}$ & $\begin{array}{l}Q_{1} \\
(W)\end{array}$ & $\begin{array}{c}Q_{2} \\
(W)\end{array}$ & $\begin{array}{l}Q_{3} \\
(W)\end{array}$ & $\begin{array}{c}\mathrm{U}_{1} \\
\left(\mathrm{~W} / \mathrm{m}^{2}\right. \\
\left.{ }^{\circ} \mathrm{C}\right)\end{array}$ & $\begin{array}{c}\mathrm{U}_{2} \\
\left(\mathrm{~W} / \mathrm{m}^{2}\right. \\
\left.{ }^{\circ} \mathrm{C}\right)\end{array}$ & $\begin{array}{c}\mathrm{U}_{3} \\
\left(\mathrm{~W} / \mathrm{m}^{2}\right. \\
\left.{ }^{\circ} \mathrm{C}\right)\end{array}$ \\
\hline 1 & 101 & 49 & 40 & 38 & 151 & 111 & 73 & 49672 & 23888 & 22308 & 1981 & 1819 & 653 \\
\hline 2 & 116 & 61 & 45 & 40 & 139 & 93 & 54 & 53382 & 21712 & 23871 & 1527 & 1381 & 792 \\
\hline 3 & 117 & 55 & 47 & 23 & 145 & 98 & 75 & 45711 & 27921 & 14081 & 1549 & 1379 & 998 \\
\hline 4 & 140 & 61 & 50 & 32 & 139 & 89 & 58 & 53937 & 27675 & 21572 & 1446 & 1322 & 931 \\
\hline 5 & 97 & 53 & 59 & 72 & 347 & 288 & 216 & 57861 & 34594 & 40124 & 2110 & 3351 & 1266 \\
\hline 6 & 137 & 53 & 43 & 46 & 347 & 305 & 259 & 56338 & 22127 & 23533 & 1618 & 1359 & 896 \\
\hline 7 & 119 & 49 & 49 & 52 & 351 & 302 & 250 & 52880 & 25789 & 30991 & 1801 & 1348 & 2373 \\
\hline 8 & 147 & 71 & 76 & 51 & 329 & 253 & 201 & 67960 & 42858 & 30870 & 1721 & 1537 & 2131 \\
\hline 9 & 109 & 58 & 56 & 47 & 242 & 186 & 139 & 54934 & 31982 & 27165 & 1852 & 1748 & 1078 \\
\hline 10 & 104 & 43 & 33 & 50 & 257 & 224 & 174 & 46720 & 18268 & 28247 & 1909 & 1399 & 1034 \\
\hline 11 & 133 & 50 & 37 & 57 & 250 & 213 & 156 & 51906 & 18481 & 33485 & 1552 & 848 & 1381 \\
\hline 12 & 111 & 41 & 42 & 47 & 259 & 217 & 170 & 44605 & 24187 & 26116 & 1401 & 1986 & 752 \\
\hline 13 & 139 & 51 & 44 & 36 & 249 & 206 & 170 & 53721 & 23101 & 21301 & 1814 & 919 & 1742 \\
\hline 14 & 126 & 56 & 39 & 36 & 122 & 83 & 48 & 47398 & 22857 & 21931 & 1543 & 1107 & 947 \\
\hline 15 & 118 & 78 & 56 & 30 & 344 & 288 & 258 & 77934 & 30810 & 16066 & 2407 & 1849 & 853 \\
\hline
\end{tabular}

Donde:

Ws $=$ Flujo de vapor

$\mathrm{W}_{1}=$ Flujo de vapor a la salida del primer efecto

$\mathrm{W}_{\mathrm{a}}=$ Flujo de jugo a la salida del primer efecto

$W_{2}=$ Flujo de vapor a la salida del segundo efecto

$W_{b}=$ flujo de jugo a las salida del segundo efecto

$W_{3}=$ Flujo de vapor a la salida del tercer efecto

$W_{\mathrm{C}}=$ Flujo de jugo a la salida del tercer efecto

$\mathrm{Q}_{1}=$ calor requerido primer efecto

$\mathrm{U}_{1}=$ coeficiente global de transferencia de calor en el primer efecto

$\mathrm{Q}_{2}=$ calor requerido segundo efecto

$\mathrm{U}_{2}=$ coeficiente global de transferencia de calor en el segundo efecto

$\mathrm{Q}_{3}=$ calor requerido tercer efecto

$\mathrm{U}_{3}=$ coeficiente global de transferencia de calor en el tercer efecto 
Conociendo el funcionamiento y las diferentes variables del proceso de evaporación de múltiple efecto, y con el fin de proponer un controlador óptimo para su funcionamiento, se formuló el modelo matemático del sistema y se resolvió el mismo usando el software Matlab.

De acuerdo a los fenómenos involucrados en la evaporación de múltiple efecto se decidió controlar dos aspectos importantes para su optimo funcionamiento: la concentración de azúcar en cada efecto (ํㅜㅅix) y el nivel en cada efecto (García et al., 2009; White et al., 2006).

\section{Control de Concentración de Azúcar en Cada Efecto (ํBrix)}

En el modelado del sistema de concentración de azúcar la variable de entrada fue la temperatura en cada efecto intercambiador $\left({ }^{\circ} \mathrm{C}\right)$ y la variable de salida fue la concentración en cada uno de los efectos (ํㅡㄹix) (Nielsen et al., 1996; Ruiz y Alvarez, 2011).

Para conocer la concentración de los jugos de caña en cada uno de los efectos se utilizó la Ec. (18).

$\mathrm{C}=(\mathrm{kg}$ de Azúcares de jugo $) /(\mathrm{kg}$ de jugo $)=\stackrel{0}{ }$ Brix

Donde, $\mathrm{C}=$ Concentración de azúcar

De acuerdo a lo anterior se realiza el balance de masa descrito por la Fig. 1.

\section{Flujoentra=Flujoquesale+Flujoacumulado}

Flujoacumulado=Flujoqueentra-Flujoquesale

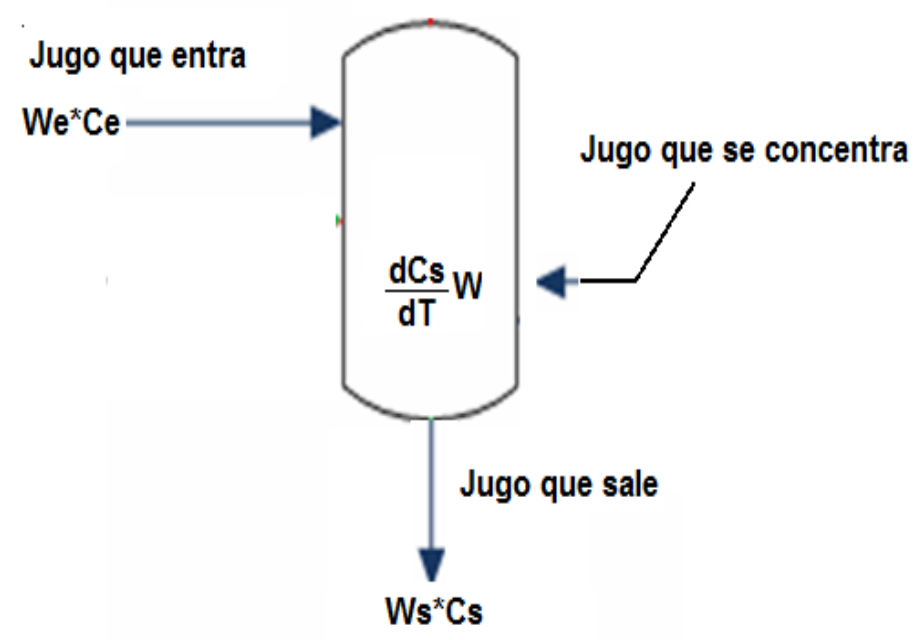

Fig. 1. Diagrama de masa y concentración

Dónde:

$\mathrm{We}=$ Masa de jugo que entra $(\mathrm{kg})$

$\mathrm{Ce}=$ Concentración de jugo entrante

$\mathrm{Ws}=$ Masa de jugo que sale $(\mathrm{kg})$

Cs = Concentración de jugo saliente

$\mathrm{W}=$ Masa que se concentra $(\mathrm{kg})$

Aplicando el balance para el jugo que se concentra se obtiene la Ec. (19).

$\frac{\mathrm{dc}}{\mathrm{dt}} \mathrm{W}=\mathrm{WeCe}-\mathrm{WsCs}$ 
Pero como la masa del jugo que entra se compone de la masa que sale del evaporador más la masa del vapor que se produce, se tiene la Ec. (20), (Gravois et al., 2009).

$W s=W e-W v$

Donde, $\mathrm{Wv}=$ Masa de vapor generado.

Remplazando la Ec. (20) en la Ec. (19) se tiene la Ec. (21).

$\frac{\mathrm{dCs}}{\mathrm{dt}} \mathrm{W}=\mathrm{WeCe}-(\mathrm{We}-\mathrm{Wv}) \mathrm{Cs}$

Reordenando los términos de la Ec. (21) se llega a la Ec. (22):

$\frac{\mathrm{dCs}}{\mathrm{dt}} \mathrm{W}=\mathrm{WeCe}-\mathrm{WeCs}+\mathrm{WvCs}$

Ahora, igualando el calor sensible y latente, y despejando $W v$ para remplazarla se obtiene la Ec. (23).

$\mathrm{Wv} \lambda=\mathrm{UA} \Delta \mathrm{T}$

Despejando Wv se llega a la Ec. (24).

$\mathrm{Wv}=\frac{\mathrm{UA \Delta T}}{\lambda}$

Haciendo el balance para el primer efecto se obtiene la Ec. (25).

$\frac{\mathrm{dCs}}{\mathrm{dt}} \rho \mathrm{V}=\mathrm{WeCe}-\mathrm{WeCs}+\frac{\mathrm{UA \Delta T}}{\lambda} \mathrm{Cs}$

Analizando la Ec. (25) la variable de entrada $\Delta T$ corresponde al calor suministrado por la caldera (en el caso del primer efecto) y la variable de salida Cs es la concentración de azúcar en la salida de los jugos. De la misma manera se determinó la concentración de jugos para el segundo efecto Ec. (26) y para el tercer efecto Ec. (27).

$\frac{\mathrm{dC} s_{2}}{\mathrm{dt}} \rho_{2} \mathrm{~V}_{2}=W \mathrm{e}_{2} \mathrm{Ce}_{2}-W \mathrm{e}_{2} \mathrm{Cs}_{2}+\frac{\mathrm{U}_{2} \mathrm{~A}_{2} \Delta \mathrm{T}_{2}}{\lambda_{2}} \mathrm{Cs}_{2}$

$\frac{\mathrm{dC} s_{3}}{\mathrm{dt}} \rho_{3} \mathrm{~V}_{3}=W \mathrm{e}_{3} \mathrm{Ce}_{3}-W \mathrm{e}_{3} \mathrm{Cs}_{3}+\frac{\mathrm{U}_{3} A_{3} \Delta \mathrm{T}_{3}}{\lambda_{3}} \mathrm{C} s_{3}$

\section{Control de Nivel en Cada Efecto}

En el modelado del control de nivel en cada efecto, la variable de entrada fue el porcentaje de apertura de válvula (a) y la variable de salida fue el nivel en cada efecto (h).

Partiendo de la ecuación de la capacitancia hidráulica Ec. (11) e inductancia hidráulica Ec. (17) y tomando como referencia los diferentes flujos, resistencias y presiones que actúan en el sistema (Fig. 2) se realiza el siguiente análisis. 


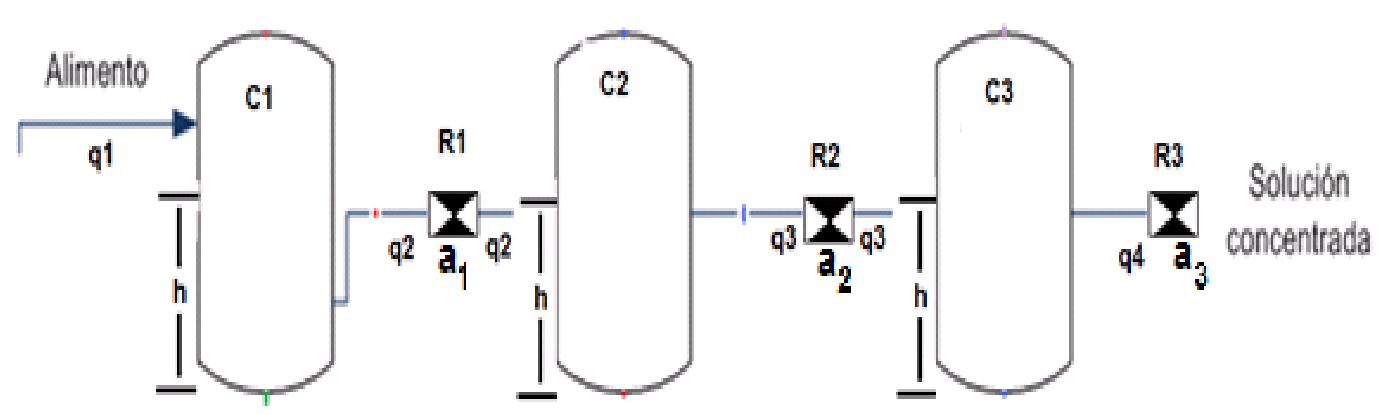

Fig. 2. Diagrama de flujos y resistencias hidráulicas

Conociendo el comportamiento previo del sistema se deduce que el movimiento de los jugos de un efecto a otro se realiza por la diferencia de presión que hay entre estos y no por una diferencia de altura, y partiendo de la ecuación de capacitancia hidráulica se obtiene la Ec. (28) (Jose, 2011).

$\mathbf{Q}_{1}-\mathbf{Q}_{2}=\frac{\mathrm{dV}}{\mathrm{dt}}$

Donde:

$\mathrm{Q}_{1}=$ Caudal $1\left(\frac{\mathbf{m}^{3}}{\mathbf{s}}\right)$

$\mathrm{Q}_{2}=$ Caudal $2\left(\frac{\mathbf{m}^{3}}{\mathbf{s}}\right)$

$V=$ Volumen $\left(\mathrm{m}^{3}\right)$

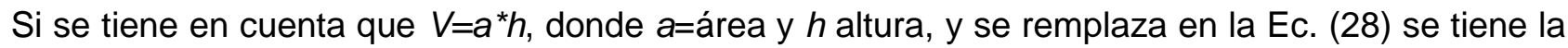
Ec. (29).

$\left(\mathbf{Q}_{1}-\mathbf{Q}_{2}\right)=\mathbf{A}_{1} \frac{\mathrm{dh}_{1}}{\mathrm{dt}}$

Ahora, si se utiliza la ecuación de resistencia hidráulica para $Q_{2}$, se llega a la Ec. (30).

$\left(\mathbf{Q}_{1}-\left(\frac{\mathbf{P}_{1}-\mathbf{P}_{2}}{\mathbf{R}_{1}}\right)\right)=\mathrm{A}_{1} \frac{\mathrm{dh}_{1}}{\mathrm{dt}}$

La Ec. (31) expresa la relación entre $R$ y el porcentaje de apertura de la válvula.

$\mathrm{R}=\frac{1}{\mathrm{a}}$

Y remplazando la Ec. (31) en la Ec. (30), se obtiene la Ec. (32) que representa el control de nivel para el primer efecto.

$Q_{1}-a_{1}\left(P_{1}-P_{2}\right)=A_{1} \frac{d h_{1}}{d t}$

Donde:

$Q_{1}=$ Caudal $1\left(\frac{\mathrm{m}^{3}}{\mathrm{~s}}\right)$

$a_{1}=$ Apertura de la válvula $\left(\frac{\mathbf{m}^{4} \mathbf{s}}{\mathrm{Kg}}\right)$

$\boldsymbol{P}_{1}=$ Presión $1(\mathrm{~N} / \mathrm{m})$ 
$P_{2}=$ Presión $2(\mathrm{~N} / \mathrm{m})$

$A_{1}=$ Área del efecto $1\left(\mathrm{~m}^{2}\right)$

$h_{1}=$ Altura del líquido $(\mathrm{m})$

De esta manera se obtiene la ecuación del efecto 1, que hace referencia a una diferencia de presión entre efectos $\left(P_{1}, P_{2}\right)$, los caudales de entrada y salida del efecto 1 ( $Q 2$ y y $\left.\left.Q\right] \_2\right)$ y la apertura de la válvula $(a)$, haciendo que fluctué la altura $(h)$ en el efecto.

De la misma manera se obtiene la ecuación del efecto 2, descrita por la Ec. (33).

$\mathbf{Q}_{2}-\mathbf{a}_{2}\left(\mathbf{P}_{2}-\mathbf{P}_{3}\right)=\mathrm{A}_{2} \frac{\mathrm{dh}_{2}}{\mathrm{dt}}$

Para el efecto 3 se realiza un análisis diferente, ya que para este caso el movimiento de las mieles concentradas se realiza por una diferencia de altura y no por una diferencia de presión, cuyo resultado es descrito por la Ec. (34).

$\mathrm{Q}_{3}-\mathrm{Q}_{4}=\mathrm{C} \frac{\mathrm{dp}}{\mathrm{dt}}$

Y remplazando $\mathrm{P}=\rho g h$ y $C={ }^{A / \rho g}$ en la Ec. (34), se obtiene la Ec. (35).

$\mathbf{Q}_{3}-\mathbf{Q}_{4}=\mathrm{A}_{3} \frac{\mathrm{dh}}{\mathrm{dt}}$

La razón por la cual sale el líquido del efecto $\left(Q_{4}\right)$, es descrita por la Ec. (36).

$\mathrm{P}=\mathrm{R}_{3} \mathrm{Q}_{4}$

La diferencia de presión para cada uno de los dos lados de la válvula es $\rho g h_{1}$ y $\rho g h_{2}$, que remplazando en la Ec. (36) se tiene la Ec. (37).

$\left(\mathrm{H}_{3}-\mathrm{H}_{4}\right) \rho_{3} \mathrm{~g}=\mathrm{R}_{3} \mathrm{Q}_{4}$

Despejando Q se llega a la Ec. (38).

$\frac{\left(\mathrm{H}_{3}-\mathrm{H}_{4}\right) \mathbf{p}_{3} \mathrm{~g}}{\mathbf{R}_{3}}=\mathbf{Q}_{4}$

Remplazando $R$ se tiene la Ec. (39).

$a_{3}\left(H_{3}-H_{4}\right) p_{3} g=Q_{4}$

Y remplazando $Q_{4}$ de la Ec. (39) se obtiene el control de nivel del efecto 3 Ec. (40).

$\mathbf{Q}_{3}-\mathrm{a}_{3}\left(\mathrm{H}_{3}-\mathrm{H}_{4}\right) \mathbf{p}_{3} \mathrm{~g}=\mathrm{A}_{3} \frac{\mathrm{dh}}{\mathrm{dt}}$

Donde:

$Q_{3}=$ Caudal3 $\left(\frac{\mathrm{m}^{3}}{\mathrm{~s}}\right)$

$a_{3}=$ Área $3\left(\frac{\mathrm{m}^{4} \mathrm{~s}}{\mathrm{~kg}}\right)$

$\boldsymbol{H}_{3}=$ Altura del efecto $3(\mathrm{~m})$

$\boldsymbol{H}_{4}=$ Atura del contenedor de mieles $(\mathrm{m})$ 
$\rho_{3}=$ Densidad del fluido $\left(\frac{\mathrm{kg}}{\mathrm{m}^{3}}\right)$

$g=\operatorname{Gravedad}\left(\frac{\mathrm{kg}^{*} \mathrm{~m}}{\mathrm{~s}^{3}}\right)$

$A_{3}=$ Área del efecto $\left(\mathrm{m}^{2}\right)$

$h_{3}=$ Altura del fluido en el efecto 3

De acuerdo a lo anterior el análisis de nivel se realizó a partir de un balance simple de los flujos que intervinieron y las caídas de presión entre cada uno de los efectos. Con respecto a las variables, las notaciones $\rho_{1}, \rho_{2}, \rho_{3}$, hacen referencia a las diferentes densidades encontrados de manera experimental, las notaciones $A_{1}, A_{2}, A_{3}$, hacen referencia a las diferentes áreas de los efectos y $g$ hace referencia al valor de la gravedad las cuales son constantes ya definidas. (Chatrattanawuth et al., 2006; Gravois et al., 2010).

\section{Modelado en lazo abierto y lazo cerrado}

El diagrama de lazo abierto del sistema representa todos los elementos que actúan en el sistema en serie, mientras que el diagrama en lazo cerrado representa una retroalimentación de la respuesta en la entrada con el fin de disminuir el error y tener control (Blanco y Peña, 2011).

En la Fig. 3 se representan las variables para el control de la concentración de azúcar.

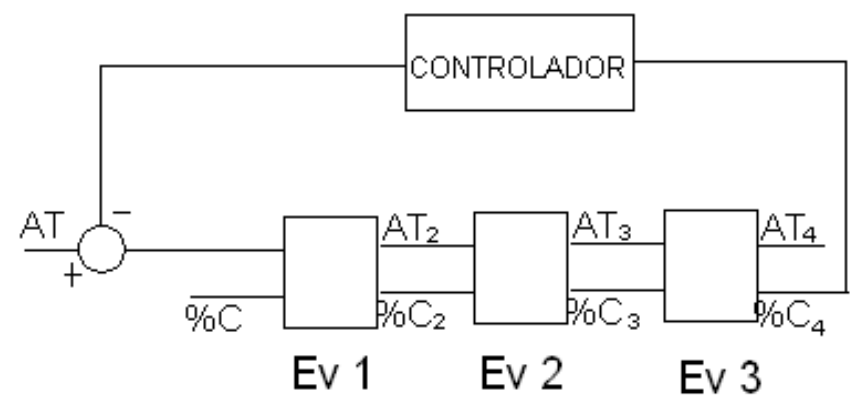

Fig. 3: Representación de las variables de entrada y salida para el control de la concentración de azúcar.

En las Fig. 4 y 5 se muestran los diagramas de bloques para el control de la concentración de azúcar, mientras que, en las Fig. 6 y 7 se muestran los diagramas de bloques en lazo abierto y cerrado respectivamente para el control de nivel.

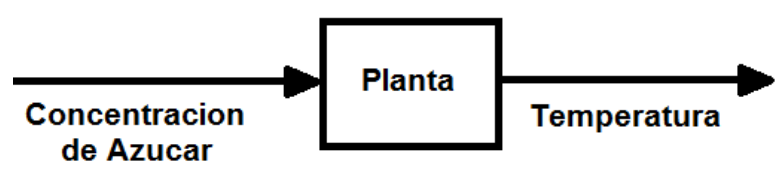

Fig.4: Diagrama de bloques en lazo abierto para el control de la concentración de azúcar

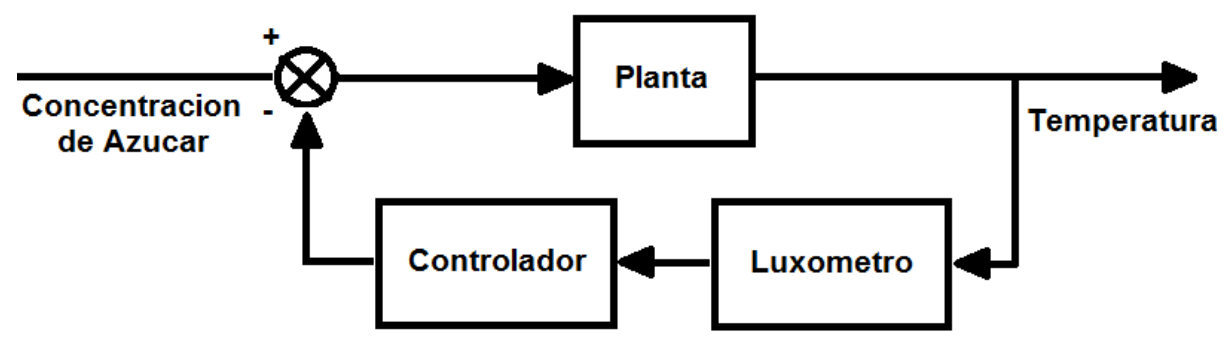

Fig. 5: Diagrama de bloques en lazo cerrado para el control de concentración de azúcar 


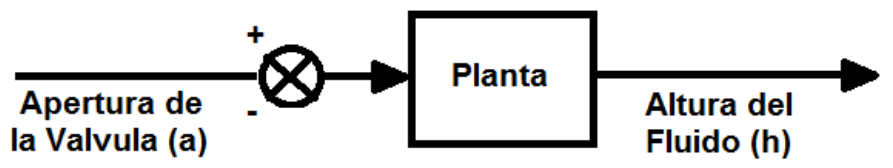

Fig. 6: Diagrama de bloques lazo abierto para el Control de nivel

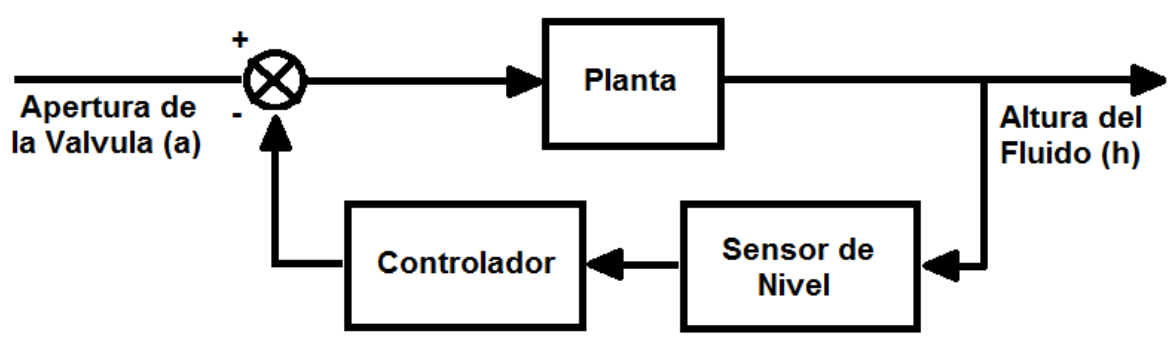

Fig. 7: Diagrama de bloques lazo cerrado para el Control de nivel Linealización del sistema de concentración

Luego de determinar el modelado del sistema de concentración de azúcar por medio de ecuaciones diferenciales, se hace necesario implementar técnicas de linealización con el fin de determinar la función de transferencia del sistema; la ecuación a linealizar y su respectiva solución luego de utilizar series de Taylor, se describen en la Ec. (41) y la Ec. (42) respectivamente.

$$
\begin{aligned}
& 13,31 \frac{d_{c}}{d_{x}}=36+0,283^{*} c^{*} \Delta T-0,05 * c \\
& 13,31 \frac{d_{c}}{d_{x}}=0,0158 \Delta T-0,0561 c
\end{aligned}
$$

Se debe tener en cuenta que el proceso de linealización de una ecuación diferencial no conserva el comportamiento de la ecuación original, debido a esto en el momento de realizar el modelado con las ecuaciones linealizadas se debe realizar una corrección con la ganancia.

\section{RESULTADOS}

Las funciones de transferencia de cada concentrador presentadas en la Fig. 8, se hallan luego de trabajar matemáticamente con las funciones de transferencia de cada componente del sistema de control del concentrador en cuestión. Luego de aplicar técnicas de reducción, se determina la función de transferencia del sistema de concentración de azúcar, descrita por la Ec. (43).

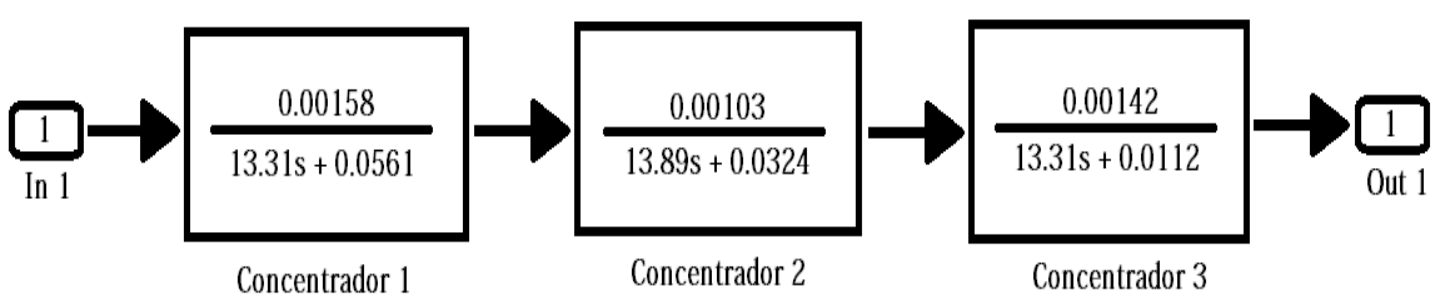

Fig. 8: Modelado del sistema de concentración completo

$H(s)=\frac{9,3 \times 10^{-13}}{s^{3}+(0,0073) s^{2}+\left(1,5 \times 10^{-7}\right) s+8,13 \times 10^{-9}}$

Luego de analizar las ecuaciones del sistema de control de nivel y del sistema incluyendo los tres efectos donde se realiza la evaporación, se obtuvo una FT de primer orden descrita por la Ec. (44). 
$\mathrm{G}(\mathrm{s})=\frac{2,757}{\mathrm{~s}+15,33}$

\section{Simulación en lazo abierto}

Para la concentración de azúcar el sistema en lazo abierto muestra estabilidad en aproximadamente 6000 segundos ( $1.6 \mathrm{~h}$ ), lo cual indica es congruente con la realidad puesto que el sistema en la práctica alcanza estabilidad en aproximadamente una hora y media, mientras que para el control de Nivel, la simulación del sistema en lazo abierto muestra una estabilidad cerca a los 30 segundos.

\section{Estabilidad del sistema}

Luego de utilizar la técnica del lugar de las raíces en Matlab para el sistema de concentración de azúcar, se encontraron tres polos al lado izquierdo del plano, lo cual indica que el sistema posee un comportamiento estable. Sin embargo, dos de los tres polos tiene una tendencia a la inestabilidad, por este motivo se planteó un controlador con el fin de garantizar la estabilidad.

Ahora para el control de nivel, de acuerdo a su FT, el sistema es estable, debido a que, el único polo que el sistema tiene se encuentra en el lado derecho del sistema y además este tiene una tendencia hacia el infinito, por consiguiente, se planteó un control proporcional.

\section{Elección y sintonía del controlador}

Para la elección y sintonía del controlador se tuvieron en cuenta dos parámetros, el máximo sobreimpulso del sistema y el tiempo de estabilización del mismo. Para el primero se determinó un valor del $10 \%$ y para el segundo un valor inferior a una hora. En la Fig. 9 se muestra la implementación del Control Proporcional Derivativo (PD) para la concentración de azúcar.

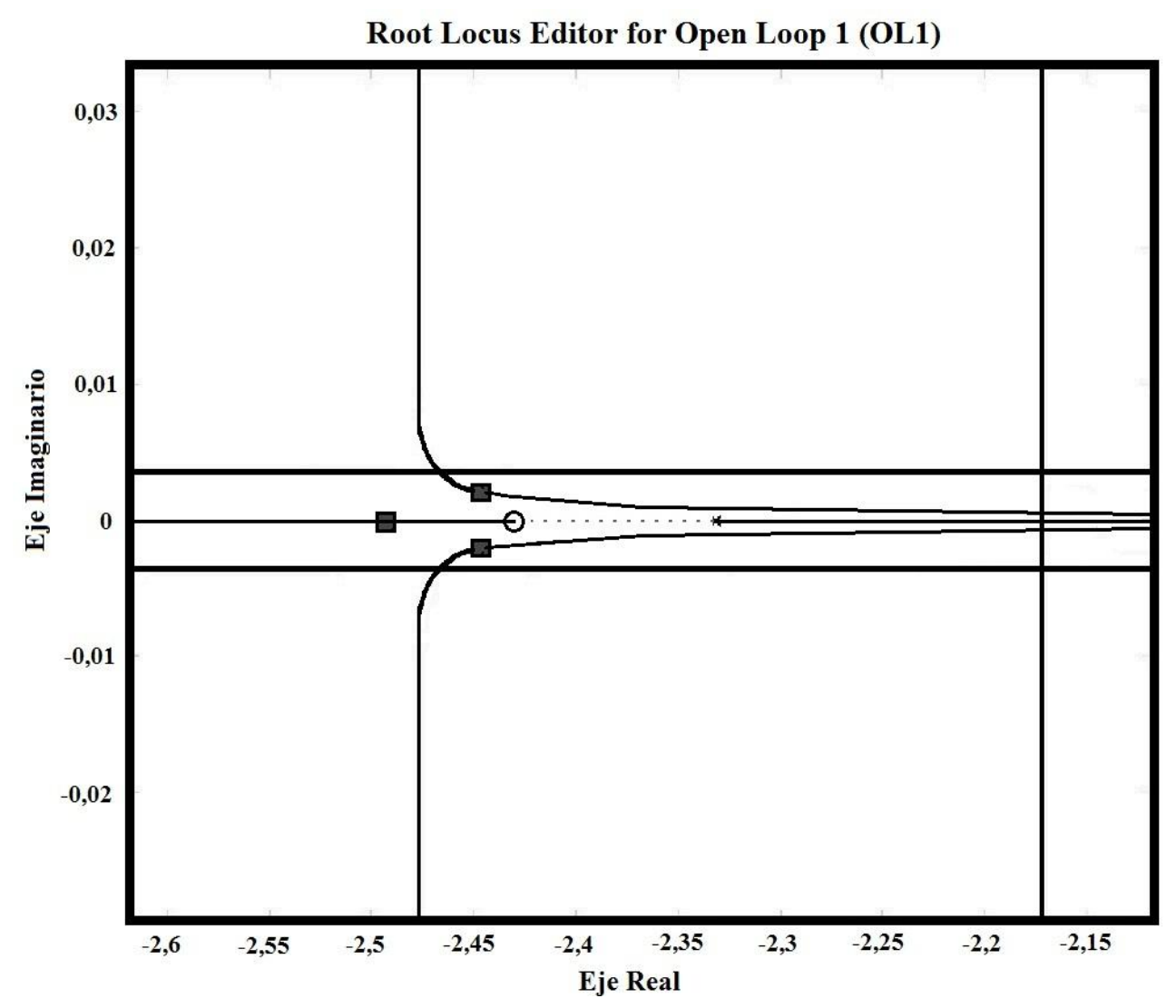

Fig.9: Implementación de control PD en el sistema de concentración de azúcar 


\section{Margen de error y correlación}

De acuerdo al modelado realizado y los datos experimentales, los cuales dependían de los instrumentos de medición, se obtuvo un margen de error del $15 \%$ aproximadamente, con un coeficiente de correlación de 0.68 .

\section{CONCLUSIONES}

Como resultado del modelo y los controladores realizados se obtuvo un sistema de evaporación de múltiple efecto más eficiente en términos de tiempos de procesamiento y optimización de recursos, analizando la dinámica del fluido entre los evaporadores, la diferencia de presión entre los evaporadores y el área del evaporador. Sin embargo, para considerar un modelo más real se debe tener en cuenta además, el cambio de densidad que se produce mientras el jugo fluye de un evaporador a otro.

\section{AGRADECIMIENTOS}

Este trabajo fue financiado a través del proyecto "Desarrollo de un sistema de evaporación y concentración de jugos de múltiple efecto para mejorar la eficiencia térmica y la productividad, y disminuir el impacto ambiental en la producción de panela", ejecutado por la Corporación colombiana de Investigación Agropecuaria - CORPOICA y financiado por el Ministerio de Agricultura y Desarrollo Rural.

\section{REFERENCIAS}

Bischoff, K.P., y K.A. Gravois. The development of new sugarcane varieties at the LSU AgCenter. J. Am. Soc. Sugar Cane Technol. 24, 142-164. (2003).

Blanco, J. M. y F. Peña, Incremento de la Eficiencia en Centrales Termoeléctricas por Aprovechamiento de los Gases de la Combustión, Información Tecnológica: 22(4), 15-22 (2011)

Chatrattanawuth, W., N. Suksariwattanagul, T. Benjanarasuth y J. Ngamwiwit, Fuzzy IPD Controller for Level Control, SICE-ICASE, 2006. International Joint Conference, 5649 - 5652, 18 al 21 de Octubre (2006).

Duran, E., "Determinación de la eficiencia energética de un sistema de evaporación de múltiple efecto empleado en la concentración de jugos de caña panelera".Trabajo de grado, Fundación Universidad de América - CORPOICA, Bogotá-Colombia, (2010).

García, H. R., Peña, A. C. y López, R. A. "Desarrollo de un sistema de evaporación y concentración de jugos de múltiple efecto para mejorar la eficiencia térmica y la productividad y disminuir el impacto ambiental en la producción de panela: tercer Informe de Avance del proyecto", CORPOICA, Bogotá-Colombia, (2009).

Gravois, K.A., y otros 7 autores, Registration of 'L 99-233' sugarcane. J. Plant Reg. 3(3), 248-252, (2009).

Gravois, K.A., y otros 10 autores, 2011. Registration of 'L 01-299' sugarcane. J. Plant Reg. 5(2), 191-195, (2010).

José, M.; William, I. Modeling and simulation of a multistage evaporator in ethanol plant using ECOSIMPRO environment, In novena conferencia internacional IEEE de Control y Automatizacion, ICCA 2011. 71 - 75, 19 al 21 de Diciembre (2011).

Mosquera, S. A., Carrera, J. E. y Villada H. S., Variables que afectan la calidad de la panela procesada en el departamento del Cauca. Biotecnología en el sector agropecuario y agroindustrial: 5(1), 17-27 (2005). 
Nielsen, K.M., T.S. Pedersen y J.F.D. Nielsen, Simulation and control of a multiple effect evaporator, Control '96, UKACC International Conference on (Conf. Publ. No. 427), 2, 1166 1171, Exeter - UK, 2 al 5 de Septiembre (1996).

Rahman, M.M. y G.W. Soon, An experimental study on condensation and evaporation heat transfer and pressure drop in microfin copper tubes, Energy and Environment, 2009. ICEE 2009. 3rd International Conference on, 104 - 109, 7 al 8 de Diciembre (2009).

Rodriguez, G., La diversification productive comme stratégie d'activation de systèmes agroalimentaires localisés: le cas de l'agro-industrie de la panela en Colombie. Cahiers Agricultures: 17(6), 572-576 (2008).

Rodriguez, G. y Requierdesjardins, D., Environmental impact of panela food-processing industry: sustainable agriculture and local agri-food production systems. International Journal of Sustainable Development: 7(3), 237-256 (2004).

Ruiz, Á. A. y H. Álvarez, Escalamiento de Procesos Químicos y Bioquímicos basado en un Modelo Fenomenológico. Información Tecnológica: 22(6), 33-52 (2011)

White, W.H., T.L. Tew, y E.P. Richard, Jr. Association of sugarcane pith, rind hardness, and fi ber with resistance to the sugarcane borer. J. Am. Soc. Sugar Cane Technol. 26, 87-100, (2006).

Zdravkovic, A., S. Turajlic y N. Marsenic, Fuzzy PI control algorithm for the multiple effect evaporation station, In Proceedings of the third IEEE conference on control applications, 1, 677 682, 24 al 26 de Agosto (1994). 
